\title{
Clinical Characteristics of Long-Term Survivors After Sorafenib Treatment for Unresectable Hepatocellular Carcinoma: A Korean National Multicenter Retrospective Cohort Study
}

\author{
Young Youn Cho, ',2 Su Jong Yu,' \\ Hye Won Lee, ${ }^{3}$ Do Young Kim, ${ }^{3}$ \\ Wonseok Kang, ${ }^{4}$ Yong-Han Paik, iD ${ }^{4}$ \\ Pil Soo Sung, ${ }^{5}$ Si Hyun Bae, ${ }^{6}$ \\ Su Cheol Park, ${ }^{7}$ Young Seok Doh,, \\ Kang Mo Kim, (iD) ${ }^{9}$ Eun Sun Jang, ${ }^{10}$ \\ In Hee Kim, "' Won Kim," \\ Yoon Jun Kim (iD) \\ 'Department of Internal Medicine and Liver \\ Research Institute, Seoul National University \\ College of Medicine, Seoul, Korea; ${ }^{2}$ Department of \\ Internal Medicine, Chung-Ang University Hospital, \\ Seoul, Korea; ${ }^{3}$ Department of Internal Medicine, \\ Yonsei University College of Medicine, Seoul, \\ Korea; ${ }^{4}$ Department of Medicine, Samsung Medical \\ Center, Sungkyunkwan University School of \\ Medicine, Seoul, Korea; ${ }^{5}$ Department of Internal \\ Medicine, Seoul St. Mary's Hospital, The Catholic \\ University of Korea College of Medicine, Seoul, \\ Korea; ${ }^{6}$ Department of Internal Medicine, \\ Eunpyeong St. Mary's Hospital, The Catholic \\ University of Korea College of Medicine, Seoul, \\ Korea; ${ }^{7}$ Department of Internal Medicine, Korea \\ Cancer Center Hospital, Korea Institute of \\ Radiological and Medical Sciences, Seoul, Korea; \\ ${ }^{8}$ Department of Internal Medicine, Eulji University \\ Hospital, Daejeon, Korea; 'Department of \\ Gastroenterology, Asan Liver Center, Asan Medical \\ Center, University of Ulsan College of Medicine, \\ Seoul, Korea; ${ }^{10}$ Departments of Internal Medicine, \\ Seoul National University Bundang Hospital, Seoul \\ National University College of Medicine, Seoul, \\ Korea; "Department of Internal Medicine, \\ Chonbuk National University Hospital, Chonbuk \\ National University Medical School, Cheongju-si, \\ Korea; ${ }^{12}$ Department of Internal Medicine, Seoul \\ Metropolitan Government Seoul National \\ University Boramae Medical Center, Seoul, Korea
}

Correspondence: Yoon Jun Kim

Department of Internal Medicine and Liver Research Institute, Seoul National University College of Medicine, I0I Daehak-no, Jongno-gu, Seoul, 03080 ( I 10-744), Korea Tel +82-2-2072-308I

Fax +82-2-743-670।

Email yoonjun@snu.ac.kr
Background/Aim: Sorafenib is the first systemic therapy for the treatment of advancedstage hepatocellular carcinoma (HCC) and progressive HCC after locoregional therapy. The aim of this study was to evaluate the prognostic factors of long-term survivors after sorafenib treatment.

Methods: This multicenter, retrospective, cohort study included 1,566 unresectable HCC patients who received sorafenib treatment between 2007 and 2014 in nine tertiary centers in Korea. The patients were classified into a long-term survivor group (survival more than two years, $n=257)$ or a control group $(n=1309)$. The primary outcomes were the prognostic factors affecting long-term survival. Secondary endpoints included time-to-progression and other safety profiles.

Results: The patients were predominantly men (83.8\%) with chronic hepatitis B $(77.3 \%)$ and Barcelona clinic of liver cancer-stage C (BCLC-C) (78.3\%). The median overall survival was 9.0 months. After treatment, eight patients $(0.4 \%)$ achieved complete response and 139 patients $(8.8 \%)$ achieved partial response according to the mRECIST criteria. The prognostic factors predicting long-term survival were metformin use (adjusted hazard ratio [aHR] = 3.464; $\mathrm{P}<0.001)$, hand-foot skin reaction $(\mathrm{aHR}=1.688 ; \mathrm{P}=0.003)$, and concomitant treatment with chemoembolization or radiotherapy $(\mathrm{aHR}=2.766$; $\mathrm{P}<0.001)$. Poor prognostic factors of long-term survival were a Child-Pugh score of $\mathrm{B}(\mathrm{HR}=0.422 ; \mathrm{P}<0.001)$, the presence of extrahepatic metastasis $(\mathrm{HR}=0.639 ; \mathrm{P}=0.005)$, main portal vein invasion $(\mathrm{HR}=0.502 ; \mathrm{P}=0.001)$, and elevated alpha-fetoprotein $(>1,000 \mathrm{ng} / \mathrm{mL} ; \mathrm{HR}=0.361 ; \mathrm{P}<$ 0.001).

Conclusion: This large, multicenter, retrospective study showed an objective response rate of $9.1 \%$ and a proportion of long-term survivors of $16.4 \%$ in Korean patients. The prognostic factors derived in our study can be used in clinical practice during sorafenib treatment.

Keywords: sorafenib, hepatocellular carcinoma, prognosis, survival

\section{Introduction}

Hepatocellular carcinoma (HCC) is the leading cause of death worldwide, and the burden of HCC is likely to increase in the future. ${ }^{1,2}$ Hepatitis B is the most common cause of liver disease and HCC in Korea. ${ }^{3}$ Although chronic liver disease patients are under a strict surveillance program, less than $30 \%$ of newly diagnosed HCC patients are detected in the curative stage. ${ }^{4}$ The five-year survival rate of 
unresectable HCC has improved recently, but the prognosis of unresectable HCC is still poor.

Sorafenib is a multi-tyrosine kinase inhibitor that impedes cell proliferation and angiogenesis. Sorafenib was approved for use in Korea based on two large-scale randomized controlled studies: the Sorafenib Hepatocellular Carcinoma Assessment Randomized Protocol (SHARP) trial and the Asia-Pacific (AP) trial. ${ }^{5,6}$ Sorafenib is currently the standard systemic therapy approved to treat $\mathrm{HCC}$ in patients with well-preserved liver function (Child-Pugh class A), advanced-stage HCC (BCLC-C), and progressive HCC after locoregional therapy. Recently, atezolizumab plus bevacizumab has demonstrated better overall survival compared to sorafenib. ${ }^{7}$ Novel treatment options for HCC have been developed, including lenvatinib for first-line treatment and regorafenib, nivolumab, cabozantinib, and ramucirumab for second-line treatment. ${ }^{8}$ However, sorafenib is still important in advanced HCC treatment because current first-line options other than atezolizumab plus bevacizumab are not superior to sorafenib, and most second-line treatments occur in a clinical setting where sorafenib treatment has failed.

Several previous studies have examined the prognostic factors that favor sorafenib treatment. A previous Japanese study descriptively analyzed profiles of patients who have survived for more than three years after sorafenib treatment that included conversion surgery following successful sorafenib treatment, additional salvage options in progressive disease, long-term sorafenib treatment, and good pre-treatment liver functions. ${ }^{9}$ Another nationwide survey in Japan examined the clinical characteristics of patients who obtained a complete response (CR) after sorafenib administration found that the prognostic factors in the CR group included female sex, low body weight $(<59 \mathrm{~kg})$, early clinical stage, and a small initial dose of sorafenib, implying that it was difficult to predict responders using patients' baseline clinical characteristics. ${ }^{10}$ Several studies focused on the on-treatment values of biomarkers such as alpha-fetoprotein (AFP), which predict sorafenib response. ${ }^{11,12}$ Moreover, previous studies have demonstrated that the development of adverse events, such as skin toxicity, diarrhea, and hypertension, was associated with improved survival after sorafenib treatment. ${ }^{13,14}$

Given the multiple treatment options for unresectable HCC, clinicians need to identify patients who will benefit from sorafenib treatment. However, little data are available regarding the clinical characteristics of good responders.
Our study aimed to evaluate the prognostic factors for long-term survival.

\section{Patients and Methods Patients}

From January 2007 to March 2014, 1,566 consecutive patients who underwent sorafenib treatment and met our inclusion/exclusion criteria were included in this study. The study protocol conformed to the ethical guidelines of the World Medical Association Declaration of Helsinki and was approved by the Institutional Review Board of Seoul National University Hospital and all the other centers. Informed consent was waived in Seoul National University and all the other centers since the research involves no more than minimal risk to the subject, the waiver will not adversely affect the rights and welfare of the subjects, and the research could not practicably be done without a waiver of consent. Non-identified information was used in order to protect the patients' confidentiality.

The inclusion criteria were as follows: (a) clinical or histological diagnosis of HCC based on the American Association for the Study of Liver Diseases guidelines; ${ }^{15,16}$ (b) unresectable HCC with BCLC stage $\mathrm{B}$ or $\mathrm{C}$; (c) tumor conditions confirmed by abdominal imaging (contrast-enhanced computed tomography [CT] and/or magnetic resonance imaging [MRI]) performed within two months before sorafenib administration; (d) treatment response evaluated by abdominal imaging (contrast-enhanced CT and/or MRI) at least once after sorafenib treatment; and (e) patients with a Child-Pugh Score of A or B. The exclusion criteria were as follows: (a) a previous history of receiving other systemic therapies (except for hepatic arterial infusion of chemotherapy, molecularly targeted agents, or immunotherapy for HCC treatment before sorafenib treatment); (b) concomitant serious medical conditions requiring medicine whose packaging recommends caution when co-administering; and (c) previous or concurrent cancer that has a primary site or histology distinct from HCC, except for cervical carcinoma in situ, treated basal cell carcinoma, superficial bladder tumors [Ta, Tis, and T1], or any cancer curatively treated less than three years prior enrolment.

\section{Sorafenib Treatment}

Patients received a starting dose of sorafenib of $400 \mathrm{mg}$ twice daily. In case of adverse events, sorafenib doses 
were adjusted according to each treating physician. Treatment was continued until tumor progression or intolerable toxicity.

\section{Endpoints and Assessment}

The index date of this study was set as the initial date of sorafenib treatment. We defined long-term survivors as patients surviving more than two years and short-term survivors as those surviving less than two years after sorafenib treatment. This period was decided based on the consensus of the multicenter study participants and previous studies.

The primary outcome of this study was the clinical characteristics of long-term survivors after sorafenib treatment. The secondary study objectives were (a) prognostic survival factors for all patients; (b) overall survival (OS, months) in all patients; and (c) safety profiles, especially off-target effects (skin toxicity, diarrhea, and arterial hypertension) in all patients.

Tumor response was assessed in each patient by the treating physician. Tumor response and disease progression were evaluated using the modified Response Evaluation Criteria in Solid Tumors (mRECIST) criteria, which assess treatment response by measuring the longest diameters of all arterial enhancing lesions. HCC showing no arterial enhancement was defined as complete responders (CR), and cases in which the viable portion was reduced by at least $30 \%$ were defined as partial responses (PR). Cases in which the viable portion increased by at least $20 \%$ or with newly developed lesions were defined as progressive disease (PD), and the remaining cases were defined as stable disease (SD). Safety profiles were evaluated according to the overall incidence of each adverse event based on the medical records. The presence or absence of off-target effects, including skin toxicities, diarrhea, and arterial hypertension, was recorded. Adverse events were graded according to Common Terminology Criteria for Adverse Events, version 4.03.

\section{Statistical Analysis}

To compare baseline characteristics, we used a two-sample $t$-test or the Kruskal-Wallis test for continuous variables and the Fisher exact test or the Chi-square test for noncontinuous variables. The Kaplan-Meier method was used to estimate survival and the Log rank test was used to compare survival curves. Univariate analysis was performed by the Cox proportional hazards model or the logistic regression model, and prognostic factors found to be significant in the univariate analysis were then combined in a multivariate Cox proportional hazards model or in a multivariate logistic regression model to obtain the optimal prediction model.

Cumulative OS and time to progression after sorafenib administration was calculated by the Kaplan-Meier method. The determination of the antitumor effect was based on objective tumor assessments documented by each physician in the medical record according to mRECIST. Additionally, the response assessment was reevaluated by an on-site radiologist according to mRECIST principles to accurately determine sorafenib effectiveness.

Univariable and multivariable Cox proportional hazards models were used to identify OS risk factors and to evaluate clinical variables in a specific subgroup, such as long-term survivors. Multivariable analysis variables were determined based on their statistical significance in the univariable analysis. To avoid multicollinearity, the forward conditional stepwise procedure was performed in the multivariable analysis. OS according to significant risk factors was compared using the Log rank test. All statistical analysis was performed using SPSS software (SPSS version 20.0; SPSS, Chicago, Ill). A two-sided $P$ value of $<0.05$ was considered statistically significant.

\section{Results}

A total of 1,566 patients were included in this study; 257 in the long-term survivor group and 1,309 patients in the short-term survivor group. The patients were predominantly men $(83.8 \%)$ with chronic hepatitis B $(77.3 \%)$ and BCLC stage C (78.3\%). The long-term survivor group had favorable baseline characteristics. A significantly higher proportion of the long-term survivors had a Child-Pugh score of $\mathrm{A}(89.9 \%$ vs $75.3 \%, \mathrm{P}<0.001$; Table 1$)$ and BCLC stage B $(34.1 \%$ vs $18.0 \%, \mathrm{P}<0.001)$ than the short-term survivor group. In addition, a significantly lower proportion of the long-term survivor group had Vp3-4 portal vein tumor thrombosis (PVTT, 15.5\% vs $31.4 \%, \mathrm{P}<0.001)$, and AFP $>1,000 \mathrm{ng} / \mathrm{mL}(17.5 \%$ vs $39.0 \%, \mathrm{P}<0.001)$. The long-term survivor group had a shorter tumor diameter $(3.64 \mathrm{~cm}$ vs $5.26 \mathrm{~cm}, \mathrm{P}<0.001)$ and fewer HCC nodules $(2.71$ vs $3.89, \mathrm{P}<0.001)$ than the short-term survivor group.

The median OS was 9.0 months (Figure 1A), and the median time to progression (TTP) was 3.0 months (Figure 1B). After treatment, eight patients $(0.4 \%)$ achieved CR and 147 patients (8.8\%) achieved PR according to the mRECIST criteria (Table 2). 
Table I Baseline Characteristics

\begin{tabular}{|c|c|c|c|}
\hline & Long Term Survivor $(\mathbf{N}=257)$ & Short Term Survivor $(\mathrm{N}=\mathrm{I}, 309)$ & P value* \\
\hline Age & $58.66 \pm 10.12$ & $57.94 \pm 10.65$ & 0.163 \\
\hline \multicolumn{4}{|l|}{ Sex } \\
\hline Male & $213(82.6)$ & $1099(84.0)$ & 0.580 \\
\hline Diabetes & $40(15.5)$ & I48 (I I.3) & 0.074 \\
\hline BMI & & & 0.007 \\
\hline$<23$ & $|2|(47.1)$ & $727(56.6)$ & \\
\hline $23-25$ & $60(23.3)$ & $282(22.0)$ & \\
\hline$>25$ & $76(29.6)$ & $275(21.4)$ & \\
\hline Etiology & & & 0.514 \\
\hline Hepatitis B & $196(76.0)$ & $1045(80.3)$ & \\
\hline Hepatitis C & $23(8.9)$ & $84(6.5)$ & \\
\hline Alcoholic liver disease & $14(5.4)$ & $70(5.4)$ & \\
\hline Non alcoholic fatty liver disease & $9(3.5)$ & $39(3.0)$ & \\
\hline Others & $16(6.2)$ & $64(4.9)$ & \\
\hline ECOG performance & & & 0.003 \\
\hline 0 & $132(55.5)$ & $577(46.9)$ & \\
\hline 1 & $102(42.9)$ & $572(46.5)$ & \\
\hline $2-4$ & $4(1.7)$ & $80(6.5)$ & \\
\hline Maximum diameter $(\mathrm{cm})$ & $3.64 \pm 3.96$ & $5.26 \pm 4.58$ & $<0.001$ \\
\hline Number of $\mathrm{HCC}$ nodules & $2.71 \pm 3.19$ & $3.89 \pm 3.68$ & $<0.001$ \\
\hline Child Pugh score & & & $<0.001$ \\
\hline$A$ & $232(89.9)$ & $986(75.3)$ & \\
\hline B & $26(10.1)$ & $323(24.7)$ & \\
\hline BCLC Stage & & & $<0.001$ \\
\hline B & $88(34.1)$ & $236(18.0)$ & \\
\hline C & $170(65.9)$ & $1073(82.0)$ & \\
\hline Extrahepatic metastasis & $127(49.2)$ & $543(4 \mid .5)$ & 0.023 \\
\hline Lymph node & $40(15.5)$ & 365 (27.9) & $<0.001$ \\
\hline Lung & $100(38.8)$ & $573(43.8)$ & 0.149 \\
\hline Bone & $20(7.8)$ & $130(9.9)$ & 0.300 \\
\hline Brain & $0(0)$ & $9(0.7)$ & 0.182 \\
\hline Adrenal & $9(3.5)$ & $55(4.2)$ & 0.731 \\
\hline Peritoneal-seeding & $15(5.8)$ & $7.6(99)$ & 0.361 \\
\hline PVTT & & & $<0.001$ \\
\hline$V_{p} I-2$ & $13(5.0)$ & $127(9.7)$ & \\
\hline$V_{p} 3$ & $17(6.6)$ & $188(14.4)$ & \\
\hline$V_{p} 4$ & $23(8.9)$ & $223(17.0)$ & \\
\hline $\operatorname{AFP}(\mathrm{ng} / \mathrm{mL})$ & & & $<0.001$ \\
\hline$<7$ & 81 (3I.5) & $221(17.0)$ & \\
\hline $7-1000$ & $|3|(5 \mid .0)$ & $572(44.0)$ & \\
\hline $1000-10,000$ & $31(12.1)$ & $265(20.4)$ & \\
\hline$>10,000$ & $14(5.4)$ & $242(18.6)$ & \\
\hline
\end{tabular}

Notes: Values are presented as mean \pm standard deviation or number (\%) unless otherwise indicated; *Quantitative variables by 2 -sample $t$-test, qualitative variables by chisquare test.

Abbreviations: BMI, body mass index; ECOG, Eastern Cooperative Oncology Group; BCLC, Barcelona Clinic Liver Cancer; PVTT, portal vein tumor thrombosis; Vpl, segmental portal vein invasion; $V_{p} 2$, right anterior/posterior portal vein; $V_{p} 3$, right/ left portal vein; $V_{p} 4$, main trunk; AFP, alpha-fetoprotein. 
A

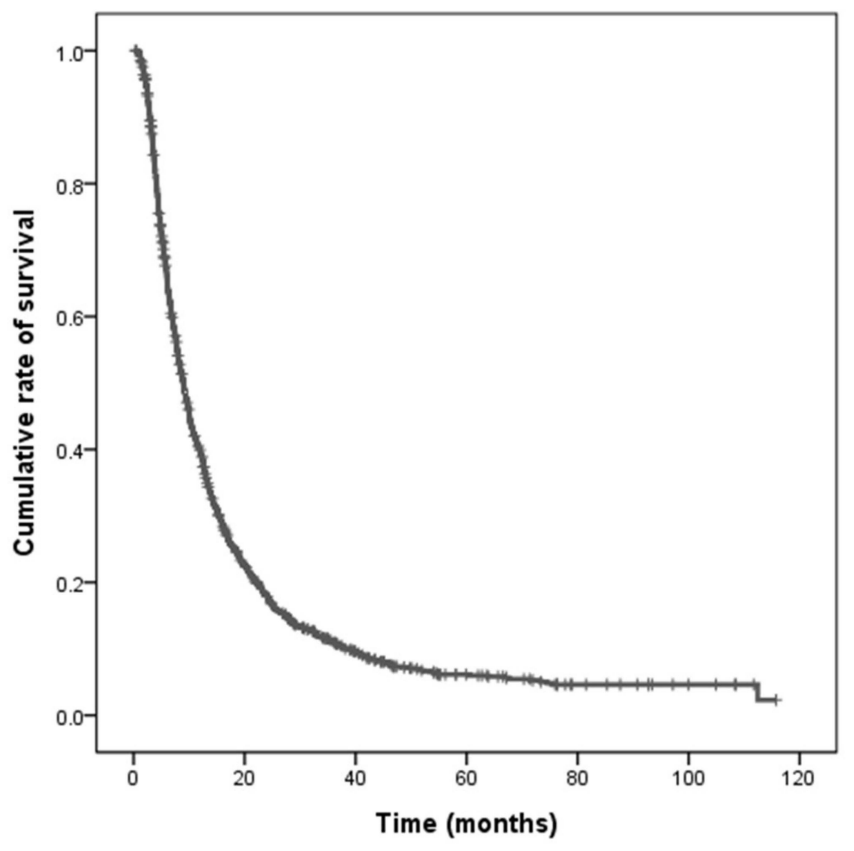

B

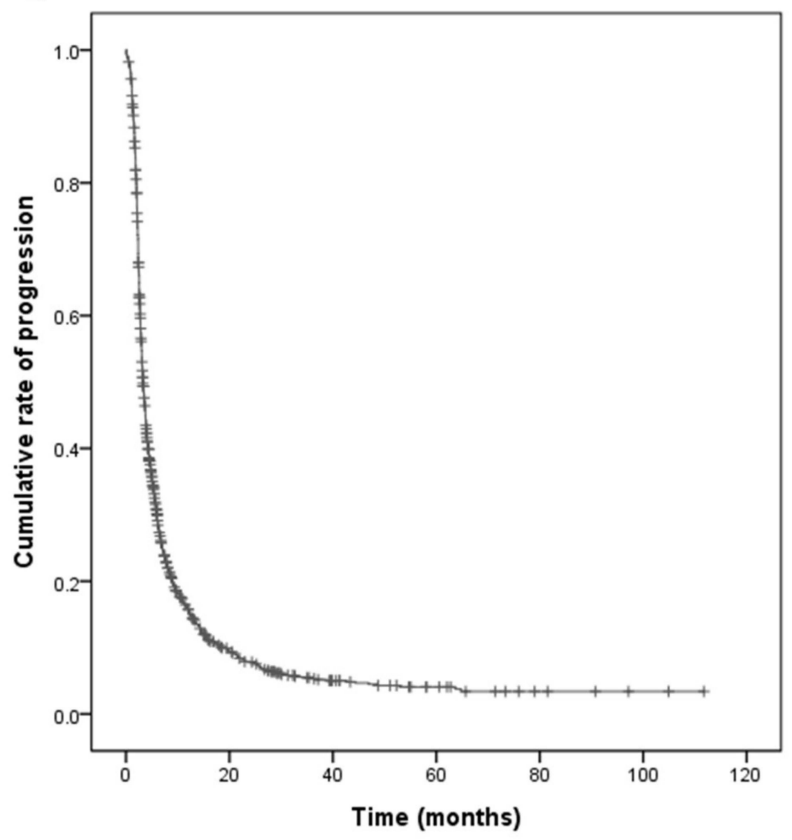

Figure I The median overall survival was 9.0 months (A) and the median time to progression was 3.0 months (B), respectively. Modified Response Evaluation Criteria in Solid Tumors criteria estimated the response.

\section{Long-Term Survivors}

The long-term survivor group included more patients treated with a lower dose of sorafenib (mean $634 \mathrm{mg}$ vs $662 \mathrm{mg}, \mathrm{P}=0.032$, Table 2) than the short-term survivor group, although the difference was minimal. Sorafenib duration was significantly longer in the long-term survivor group (median 8.6 months vs 3.0 months, $\mathrm{P}<0.001$ ) than in the short-term survivor group. Only the long-term survivor group included patients who achieved CR. Moreover, the long-term survivor group had a higher proportion of PR (14.7\% vs $7.7 \%)$ and SD (62.0\% vs $35.3 \%)$ than the short-term survivor group. In addition, SD duration was significantly longer in the long-term survivor group (46.7 months vs 14.5 months) than in the shortterm survivor group. There was a higher proportion of patients with a decrease in AFP of more than $20 \%$ at the first evaluation in the long-term survivor group $(41.4 \%$ vs $25.1 \%$ ) than in the short-term survivor group.

Eight patients achieved CR after sorafenib treatment (Supplemental Table 1). Some patients had a favorable tumor burden. Three (37.5\%) patients had Vp3-4 PVTT, two $(25.0 \%)$ had extrahepatic metastasis, and three (37.5\%) received concomitant trans-arterial chemoembolization (TACE) treatment. We compared the clinical characteristics of the patients achieving PR and the number of HCC (2.70 vs 3.83, respectively; Supplemental Table 2) and the proportion of AFP $>1,000 \mathrm{ng} / \mathrm{mL}$ (43.6\% vs 23.2 , respectively) was different. Patients with Child Pugh B showed unfavorable baseline characteristics compared to patients with Child Pugh A (Supplemental Table 3). And patients with initial AFP > 1,000 ng/mL showed unfavorable baseline characteristics compared to patients with initial AFP $\leq 1,000 \mathrm{ng} / \mathrm{mL}$ (Supplemental Table 4). There were more concomitant treatment, and less extrahepatic metastasis in patients with decrease in AFP of more than $20 \%$ at the first evaluation compared to patients with AFP decrease less than $20 \%$ or AFP increase at the first evaluation (Supplemental Table 5).

In the multivariable analysis, the prognostic factors predicting long-term survival include metformin use (adjusted hazard ratio $[\mathrm{aHR}]=3.464 ; 95 \%$ confidence interval [CI], 1.875-6.402; $P<0.001$; Table 3), dermatological adverse reactions $(\mathrm{aHR}=1.688 ; 95 \% \mathrm{CI}, 1.196-$ 2.384; $\mathrm{P}=0.003$; Table 3 ), a high body mass index ( $\mathrm{aHR}=$ 1.577; 95\% CI, 1.102-2.258; $\mathrm{P}=0.043$; Table 3), and concomitant treatment with chemoembolization or radiotherapy (aHR $=2.766 ; 95 \%$ CI, 2.016-4.213; P < 0.001; Table 3). Poor prognostic factors for long-term survival were a high Child-Pugh score $(\mathrm{aHR}=0.422 ; 95 \% \mathrm{CI}$, 
Table 2 Clinical Characteristics of Long-Term Survivors Compared to Short-Term Survivors

\begin{tabular}{|c|c|c|c|}
\hline & Long-Term Survivor $(\mathbf{N}=\mathbf{2 5 7})$ & Short-Term Survivor $(\mathrm{N}=1,309)$ & P value* \\
\hline Sorafenib dose (mg) & 634 & 662 & 0.032 \\
\hline Treatment duration of sorafenib (median, mo) & 8.6 & 3.0 & $<0.001$ \\
\hline Best overall response & & & $<0.001$ \\
\hline CR & $8(3.5)$ & $0(0)$ & \\
\hline PR & $38(14.7)$ & 101 (7.7) & \\
\hline SD & $160(62.0)$ & $462(35.3)$ & \\
\hline PD & $51(19.8)$ & $746(57.0)$ & \\
\hline SD duration (month) & $11.18 \pm 11.71$ & $4.17 \pm 3.23$ & $<0.001$ \\
\hline PR duration (month) & $9.53 \pm 14.55$ & $3.39 \pm 2.88$ & 0.014 \\
\hline CR duration (month) & $13.63 \pm 15.9 \mid$ & & \\
\hline Ratio of AFP at first evaluation & & & $<0.001$ \\
\hline$<80 \%$ & $103(4 \mid .4)$ & $292(25.1)$ & \\
\hline $80-120 \%$ & $64(25.7)$ & $248(21.3)$ & \\
\hline$>120 \%$ & $82(32.9)$ & $622(53.5)$ & \\
\hline Concomittent treatment & $71(27.5)$ & $158(\mid 2.1)$ & $<0.001$ \\
\hline Salvage treatment & $150(58.1)$ & $420(32.1)$ & $<0.001$ \\
\hline Sorafenib as first line treatment & $65(25.2)$ & 407 (3I.I) & 0.059 \\
\hline
\end{tabular}

Notes: Values are presented as mean \pm standard deviation or number (\%) unless otherwise indicated; *Quantitative variables by 2 -sample $t$-test, qualitative variables by chisquare test.

Abbreviations: CR, complete response; PR, partial response; SD, stable disease; PD, progressive disease; AFP, alpha-fetoprotein

$0.264-0.674 ; P<0.001$; Table 3$)$, the presence of extrahepatic metastasis $(\mathrm{HR}=0.639 ; 95 \% \mathrm{CI}, 0.469-0.871 ; \mathrm{P}=$ 0.005 ; Table 3$)$, main portal vein invasion $(\mathrm{aHR}=0.502$; 95\% CI, 0.296-0.853; P =0.001), and AFP levels $>1,000$ $\mathrm{ng} / \mathrm{mL}(\mathrm{aHR}=0.361 ; 95 \% \mathrm{CI}, 0.224-0.583 ; \mathrm{P}<0.001$; Table 3).

The clinical characteristics of patients treated concomitantly with chemoembolization or radiotherapy were comparable to those of patients treated with sorafenib monotherapy (Supplemental Table 6). The number of HCC nodules was smaller in the concomitant treatment group than in the group without concomitant treatment (2.45 vs $3.83, \mathrm{P}=0.017$ ).

\section{Survival}

The median OS was 9.0 months (Figure 1A), and the median time to progression was 3.0 months. Patients with overall adverse event grades of 2-4 or $0-1$ had comparable survival; however, patients with dermatologic adverse event grades of 2-4 had better median survival than patients with dermatologic adverse event grades of 0-1 (median 10.7 months vs 8.5 months, respectively, $\mathrm{P}=0.002$ by Log rank test). Patients who underwent concomitant treatment had better median survival rates than those without concomitant treatment (median 13.2 months vs 8.3 months, respectively, $\mathrm{P}<0.001$ by $\log$ rank test). Patients who received salvage treatment after sorafenib treatment failure had better median survival than those who did not receive salvage treatment (median 13.5 months vs 6.7 months, respectively, $\mathrm{P}<0.001$ by Log rank test).

In the multivariable analysis, the prognostic factors predicting OS and long-term survival were comparable (Supplemental Table 7). The only difference was that the body mass index was not a prognostic factor in predicting OS.

\section{Safety}

Overall adverse events over grade 2 were similar between the long-term survivor and the short-term survivor groups (41.9\% vs 37.7\%, respectively, $\mathrm{P}=0.205$; Table 4). However, there were more adverse events over grade 3 $(7.8 \%$ vs $12.7 \%$, respectively, $\mathrm{P}=0.025)$ and fewer 
Table 3 Prognostic Factors for Long-Term Survivors (Survival > 2 Years) After Sorafenib Treatment

\begin{tabular}{|c|c|c|c|c|}
\hline Variable & Univariate HR $(95 \% \mathrm{Cl})$ & $P$ value & Multivariate HR $(95 \% \mathrm{Cl})$ & P value* \\
\hline Age & $1.006(0.994-1.019)$ & 0.319 & & \\
\hline \multicolumn{5}{|l|}{ Sex } \\
\hline Female & I.I06 (0.776-I.575) & 0.578 & & \\
\hline BMI & & 0.007 & & 0.043 \\
\hline$<23$ & I & & 1 & \\
\hline $23-25$ & $1.278(0.911-1.794)$ & & I.I25 (0.765-I.654) & \\
\hline $25-30$ & $1.660(1.207-2.284)$ & & 1.577 (I.102-2.258) & \\
\hline ECOG & & 0.006 & & 0.027 \\
\hline 0 & I & & I & \\
\hline 1 & $0.779(0.587-1.035)$ & & $0.766(0.563-1.044)$ & \\
\hline $2-4$ & $0.219(0.079-0.607)$ & & $0.286(0.099-0.828)$ & \\
\hline Child Pugh score & & $<0.001$ & & $<0.001$ \\
\hline A & I & & I & \\
\hline B & $0.342(0.224-0.523)$ & & $0.422(0.264-0.674)$ & \\
\hline Extrahepatic metastasis & $0.731(0.559-0.956)$ & 0.022 & $0.639(0.469-0.87 I)$ & 0.005 \\
\hline PVTT & & $<0.001$ & & 0.001 \\
\hline None & 1 & & 1 & \\
\hline$V_{p} I-2$ & $0.385(0.213-0.695)$ & & $0.483(0.254-0.920)$ & \\
\hline$V_{p} 3$ & $0.340(0.202-0.572)$ & & $0.396(0.223-0.700)$ & \\
\hline$V_{p} 4$ & $0.388(0.246-0.612)$ & & $0.502(0.296-0.853)$ & \\
\hline AFP $(\mathrm{ng} / \mathrm{mL})$ & & $<0.001$ & & $<0.001$ \\
\hline$<7$ & I & & $\mathrm{I}$ & \\
\hline $7-1,000$ & $0.514(0.379-0.698)$ & & $0.568(0.404-0.799)$ & \\
\hline $1,000-10,000$ & $0.315(0.206-0.482)$ & & $0.36 \mathrm{I}(0.224-0.583)$ & \\
\hline$>10,000$ & $0.156(0.087-0.277)$ & & $0.211(0.112-0.397)$ & \\
\hline Diabetes & $1.438(0.985-2.099)$ & 0.060 & & \\
\hline Metformin Use & $3.202(1.856-5.526)$ & $<0.001$ & $3.464(1.875-6.402)$ & $<0.001$ \\
\hline Statin Use & $1.136(0.650-1.987)$ & 0.655 & & \\
\hline Adverse event $\mathrm{Gr} 2-4$ versus. Gr $0-1$ & $1.192(0.908-1.563)$ & 0.205 & & \\
\hline Dermatologic adverse event Gr 2-4 versus. Gr 0-I & $1.813(1.335-2.461)$ & $<0.001$ & $1.688(1.196-2.384)$ & 0.003 \\
\hline Gastroenterologic adverse event Gr $2-4$ versus. Gr $0-1$ & $1.378(0.910-2.085)$ & 0.130 & & \\
\hline Concomittent treatment & $2.766(2.009-3.808)$ & $<0.001$ & $2.915(2.016-4.213)$ & $<0.001$ \\
\hline
\end{tabular}

Note: *By logistic regression.

Abbreviations: BMI, body mass index; ECOG, Eastern Cooperative Oncology Group; PVTT, portal vein tumor thrombosis; AFP, alpha-fetoprotein; Gr, grade.

dermatologic adverse events $(28.3 \%$ vs $17.9 \%$, respectively, $\mathrm{P}<0.001)$ in the short-term survivor group than in the long-term survivor group.

\section{Discussion}

This is a large retrospective cohort study of 1,566 $\mathrm{HCC}$ patients who underwent sorafenib treatment in a hepatitis
B endemic area. The median OS was 9.0 months. After treatment, $9.1 \%$ of patients achieved an objective response according to mRECIST criteria. The prognostic factors favoring long-term survival were metformin use, dermatologic adverse reactions, and concomitant treatment with chemoembolization or radiotherapy. Poor prognostic factors of long-term survival were a high Child-Pugh score, 
Table 4 Safety Profiles After Sorafenib Treatment

\begin{tabular}{|l|l|l|l|}
\hline & Long-Term Survivor (N=257) & Short-Term Survivor (N=I,309) & P value* \\
\hline AE all grades (\%) & $176(68.2)$ & $846(64.6)$ & 0.269 \\
\hline AE grade 2-4 (\%) & $108(4 I .9)$ & $493(37.7)$ & 0.205 \\
\hline AE grade 3-4 (\%) & $20(7.8)$ & $166(12.7)$ & 0.025 \\
\hline Dermatologic adverse event grade 2-4 (\%) & $73(28.3)$ & $234(17.9)$ & $<0.00 I$ \\
\hline Gastroenterologic adverse event grade 2-4 (\%) & $32(12.4)$ & $122(9.3)$ & 0.128 \\
\hline Hypertension grade 2-4 (\%) & 0 & $2(0.2)$ & 0.530 \\
\hline
\end{tabular}

Note: *By chi-square test.

Abbreviation: $\mathrm{AE}$, adverse events.

the presence of extrahepatic metastasis, main portal vein invasion, and elevated AFP levels.

Important data regarding prognosis after sorafenib treatment comes from the SHARP and AP studies. ${ }^{17}$ A recent subgroup analysis of these studies showed that the presence of macroscopic vascular invasion, high AFP levels, and a high neutrophil-to-lymphocyte ratio were prognostic factors in sorafenib treatment. ${ }^{17}$ Several studies suggested that dermatologic adverse events, diarrhea, Child-Pugh score, BCLC stage, sorafenib dose, etiology, metformin treatment, and extrahepatic spread may be prognostic factors. ${ }^{18}$ In our study, well-known prognostic factors such as the Child-Pugh score, the extent of PVTT, high AFP levels, and poor performance were prognostic factors for long-term survival. Previous research indicates that extrahepatic metastasis is an important prognostic factor in sorafenib treatment, ${ }^{19}$ and our study supports that this is an independent negative prognostic factor of long-term survival.

There have been several biomarker studies on sorafenib treatment. Our work validates that the AFP response during sorafenib treatment can predict prognosis. ${ }^{11}$ In previous research, early AFP increase four weeks after sorafenib treatment predicted progressive disease and poor survival. ${ }^{12}$ The fact that the AFP response rate was significantly higher in the long-term survivor group than in the short-term survivor group can be applied in clinical practice. Previous biomarker studies have also evaluated blood inflammatory markers, such as changes in the neutrophil-to-lymphocyte ratio, to predict prognosis. ${ }^{17,20,21}$ There also have been several molecular biomarkers studies. High ACSL4 expression, a positive-activating enzyme of ferroptosis, could predict a good response to sorafenib. ${ }^{22}$ A recent study showed that stratification using VEGFR2, PDGFRB, c-KIT, c-RAF, EGFR, mTOR, and FGFR1 gene expression could predict sorafenib response. ${ }^{23}$ However, these studies could not be evaluated due to the retrospective nature of our study and a lack of laboratory data.

We found that concomitant treatment with TACE or RT predicts long-term survival and OS. The Global Investigation of therapeutic DEcisions in hepatocellular carcinoma and Of its treatment with sorafeNib (GIDEON) study ${ }^{24}$ demonstrated the safety of combining TACE with sorafenib, and several retrospective studies have shown the efficacy of combined TACE and sorafenib treatments in survival benefit and tumor response. ${ }^{25-27}$ However, recent clinical trials comparing the efficacy of sorafenib plus TACE with TACE alone in intermediate $\mathrm{HCC}^{28}$ and a Phase 3 trial comparing the same in advanced $\mathrm{HCC}^{29}$ indicate that the combination treatment did not improve OS. Although a recent Phase 3 clinical trial has shown that TACE in combination with sorafenib has no survival that benefits patients, subgroup analysis has evaluated that receiving more than two TACE sessions showed better OS in the combination group. ${ }^{29}$ External beam radiotherapy and brachytherapy using Y-90 are widely used to treat unresectable $\mathrm{HCC}^{30}$ A recent Korean randomized control study showed that TACE combined with radiotherapy resulted in better OS than sorafenib in HCC with portal vein invasion. ${ }^{31}$ And a recent Japanese randomized trial has shown that TACE plus sorafenib significantly improved PFS over TACE, implying the potential of the combination treatment. ${ }^{32}$ Our study showed that concomitant locoregional treatment is a positive prognostic factor for long-term survival. 
Further studies might elucidate a certain subgroup favoring the combination treatment which we could not specify.

Research suggests that metformin use can reduce resistance to sorafenib and improve OS. ${ }^{33,34}$ Metformin is associated with improved survival in other treatments for HCC. ${ }^{35,36}$ Metformin can also reduce HCC development, and recurrence after curative treatments. ${ }^{37,38}$ Our study showed that metformin use was associated with long-term survival even after adjusting for diabetes. These findings cannot justify the routine use of metformin, but we can include metformin in order of priority when treating cases of $\mathrm{HCC}$ with pre-existing diabetes. A recent meta-analysis showed that dermatologic adverse events are a beneficial indicator for HCC patients receiving sorafenib, and several reports suggest that hypertension and gastrointestinal adverse events are as well. ${ }^{39}$ However, the results of our study indicate that only dermatologic adverse events were associated with long-term survival after sorafenib treatment.

A strength of our study is that it is a large, multicenter cohort study that provides information in a setting where hepatitis B is the predominant cause of liver cirrhosis. The efficacy of sorafenib was significantly different between etiologies, and hepatitis B was a poor prognostic factor. ${ }^{40}$ It is important to study the efficacy of sorafenib in different etiologies. Our study is the largest retrospective study evaluating the outcomes of sorafenib treatment in an HBV endemic area. A limitation of our study is its retrospective nature, although this design allows us to determine the appropriate associations. Ongoing or future clinical trials with a sufficient number of patients are warranted.

In conclusion, this large multicenter retrospective study of Korean patients demonstrated an objective response of $9.1 \%$ and a proportion of long-term survivors of $16.4 \%$. The prognostic factors derived in our study can be used in clinical practice during sorafenib treatment.

\section{Highlights}

This multicenter, retrospective, cohort study included 1,566 unresectable HCC Korean patients who received sorafenib treatment. The objective response rate was $9.1 \%$, and the proportion of long-term survivors was $16.4 \%$. The prognostic factors predicting long-term survival were metformin use, hand-foot skin reaction, and concomitant treatment with chemoembolization or radiotherapy. Poor prognostic factors of long-term survival were a Child-Pugh score of B, the presence of extrahepatic metastasis, main portal vein invasion, and elevated alphafetoprotein levels.

\section{Author Contributions}

Guarantor of article: YJK. Specific author contributions: YYC and YJK designed the study and wrote the paper. SJY contributed to the design of the study. YYC, HWL, DYK, WK, YHP, PSS, SHB, SCP, YSD, KMK, ESJ, IHK, and WK collected and analyzed the data. All authors contributed to data analysis, drafting or revising the article, have agreed on the journal to which the article will be submitted, gave final approval of the version to be published, and agree to be accountable for all aspects of the work.

\section{Funding}

This research was supported by a grant from Bayer Pharmaceuticals Company.

\section{Disclosure}

The authors report no conflicts of interest regarding this article.

\section{References}

1. Torre LA, Bray F, Siegel RL, Ferlay J, Lortet-Tieulent J, Jemal A. Global cancer statistics, 2012. CA Cancer J Clin. 2015;65:87-108. doi: $10.3322 /$ caac. 21262

2. Venook AP, Papandreou C, Furuse J, de Guevara LL. The incidence and epidemiology of hepatocellular carcinoma: a global and regional perspective. Oncologist. 2010;15(Suppl 4):5-13. doi:10.1634/theoncologist.2010-S4-05

3. Kim BH, Park JW. Epidemiology of liver cancer in South Korea. Clin Mol Hepatol. 2018;24:1-9. doi:10.3350/cmh.2017.0112

4. European Association for the Study of the Liver. EASL clinical practice guidelines: management of hepatocellular carcinoma. J Hepatol. 2018;69:182-236.

5. Llovet JM, Ricci S, Mazzaferro V, et al. Sorafenib in advanced hepatocellular carcinoma. $N$ Engl J Med. 2008;359:378-390. doi:10.1056/NEJMoa0708857

6. Cheng AL, Kang YK, Chen Z, et al. Efficacy and safety of sorafenib in patients in the Asia-Pacific region with advanced hepatocellular carcinoma: a Phase III randomised, double-blind, placebo-controlled trial. Lancet Oncol. 2009;10(1):25-34. doi:10.1016/S1470-2045(08)70285-7

7. Finn RS, Qin S, Ikeda M, et al. Atezolizumab plus bevacizumab in unresectable hepatocellular carcinoma. $N$ Engl $J$ Med. 2020;382:1894-1905. doi:10.1056/NEJMoa1915745

8. Omata M, Cheng AL, Kokudo N, et al. Asia-Pacific clinical practice guidelines on the management of hepatocellular carcinoma: a 2017 update. Hepatol Int. 2017;11:317-370.

9. Tanaka K, Shimada M, Kudo M. Characteristics of long-term survivors following sorafenib treatment for advanced hepatocellular carcinoma: report of a workshop at the 50th Annual Meeting of the Liver Cancer Study Group of Japan. Oncology. 2014;87(Suppl 1):104-109. doi:10.1159/000368153

10. Shiba S, Okusaka T, Ikeda M, Saito H, Ichida T. Characteristics of 18 patients with hepatocellular carcinoma who obtained a complete response after treatment with sorafenib. Hepatol Res. 2014;44:1268-1276. doi:10.1111/hepr.12297

11. Personeni N, Bozzarelli S, Pressiani T, et al. Usefulness of alpha-fetoprotein response in patients treated with sorafenib for advanced hepatocellular carcinoma. J Hepatol. 2012;57:101-107. doi:10.1016/j.jhep.2012.02.016 
12. Nakazawa T, Hidaka H, Takada $J$, et al. Early increase in alpha-fetoprotein for predicting unfavorable clinical outcomes in patients with advanced hepatocellular carcinoma treated with sorafenib. Eur J Gastroenterol Hepatol. 2013;25:683-689. doi:10.1097/MEG.0b013e32835d913b

13. Di Costanzo GG, de Stefano G, Tortora R, et al. Sorafenib off-target effects predict outcomes in patients treated for hepatocellular carcinoma. Future Oncol. 2015;11:943-951. doi:10.2217/fon.14.291

14. Reig M, Torres F, Rodriguez-Lope C, et al. Early dermatologic adverse events predict better outcome in HCC patients treated with sorafenib. $J$ Hepatol. 2014;61:318-324. doi:10.1016/j. jhep.2014.03.030

15. Kim TH, Kim SY, Tang A, Lee JM. Comparison of international guidelines for noninvasive diagnosis of hepatocellular carcinoma: 2018 update. Clin Mol Hepatol. 2019;25:245-263. doi:10.3350/ cmh.2018.0090

16. Marrero JA, Kulik LM, Sirlin CB, et al. Diagnosis, staging, and management of hepatocellular carcinoma: 2018 practice guidance by the American association for the study of liver diseases. Hepatology. 2018;68:723-750. doi:10.1002/hep.29913

17. Bruix J, Cheng AL, Meinhardt G, Nakajima K, De Sanctis Y, Llovet J. Prognostic factors and predictors of sorafenib benefit in patients with hepatocellular carcinoma: analysis of two phase III studies. $J$ Hepatol. 2017;67:999-1008. doi:10.1016/j. jhep.2017.06.026

18. Marisi G, Cucchetti A, Ulivi P, et al. Ten years of sorafenib in hepatocellular carcinoma: are there any predictive and/or prognostic markers? World J Gastroenterol. 2018;24:4152-4163. doi:10.3748/ wjg.v24.i36.4152

19. Yoo JJ, Chung GE, Lee JH, et al. Sub-classification of advanced-stage hepatocellular carcinoma: a cohort study including 612 patients treated with sorafenib. Cancer Res Treat. 2018;50:366-373. doi:10.4143/crt.2017.126

20. Lué A, Serrano MT, Bustamante FJ, et al. Neutrophil-to-lymphocyte ratio predicts survival in European patients with hepatocellular carcinoma administered sorafenib. Oncotarget. 2017;8:103077-103086. doi:10.18632/oncotarget.21528

21. Wong L, Bozhilov K, Hernandez B, et al. Underlying liver disease and advanced stage liver cancer are associated with elevated neutrophil-lymphocyte ratio. Clin Mol Hepatol. 2019;25:305-316. doi:10.3350/cmh.2019.0004

22. Feng J, Lu PZ, Zhu GZ, et al. ACSL4 is a predictive biomarker of sorafenib sensitivity in hepatocellular carcinoma. Acta Pharmacol Sin. 2021;42:160-170. doi:10.1038/s41401-020-0439-x

23. Kim CM, Hwang S, Keam B, et al. Gene signature for sorafenib susceptibility in hepatocellular carcinoma: different approach with a predictive biomarker. Liver Cancer. 2020;9:182-192. doi:10.1159/ 000504548

24. Geschwind JF, Kudo M, Marrero JA, et al. TACE treatment in patients with sorafenib-treated unresectable hepatocellular carcinoma in clinical practice: final analysis of GIDEON. Radiology. 2016;279:630-640. doi:10.1148/radiol.2015150667

25. Varghese J, Kedarisetty C, Venkataraman J, et al. Combination of TACE and sorafenib improves outcomes in BCLC stages B/C of hepatocellular carcinoma: a single centre experience. Ann Hepatol. 2017;16:247-254. doi:10.5604/16652681.1231585

26. Wu FX, Chen J, Bai T, et al. The safety and efficacy of transarterial chemoembolization combined with sorafenib and sorafenib mono-therapy in patients with BCLC stage B/C hepatocellular carcinoma. BMC Cancer. 2017;17:645. doi:10.1186/s12885-017$3545-5$
27. Lee S, Kim BK, Kim SU, et al. Efficacy of sorafenib monotherapy versus sorafenib-based loco-regional treatments in advanced hepatocellular carcinoma. PLoS One. 2013;8:e77240. doi:10.1371/journal. pone. 0077240

28. Lencioni R, Llovet JM, Han G, et al. Sorafenib or placebo plus TACE with doxorubicin-eluting beads for intermediate stage $\mathrm{HCC}$ : the SPACE trial. J Hepatol. 2016;64:1090-1098. doi:10.1016/j. jhep.2016.01.012

29. Park JW, Kim YJ, Kim DY, et al. Sorafenib with versus without concurrent conventional transarterial chemoembolization (cTACE) in patients with advanced hepatocellular carcinoma (HCC): results from a multicenter, open-label, randomized, controlled phase III STAH trial. J Hepatol. 2018;68:S2. doi:10.1016/S0168-8278(18)30222-8

30. Yoon HI, Seong J. Multimodality treatment involving radiotherapy for advanced liver-confined hepatocellular carcinoma. Oncology. 2014;87(Suppl 1):90-98. doi:10.1159/000368151

31. Yoon SM, Ryoo BY, Lee SJ, et al. Efficacy and safety of transarterial chemoembolization plus external beam radiotherapy vs sorafenib in hepatocellular carcinoma with macroscopic vascular invasion: a randomized clinical trial. JAMA Oncol. 2018;4:661-669. doi:10.1001/jamaoncol.2017.5847

32. Kudo M, Ueshima K, Ikeda M, et al. Randomised, multicentre prospective trial of transarterial chemoembolisation (TACE) plus sorafenib as compared with TACE alone in patients with hepatocellular carcinoma: TACTICS trial. Gut. 2020;69:1492-1501. doi:10.1136/ gutjnl-2019-318934

33. Casadei Gardini A, Faloppi L, De Matteis S, et al. Metformin and insulin impact on clinical outcome in patients with advanced hepatocellular carcinoma receiving sorafenib: validation study and biological rationale. Eur J Cancer. 2017;86:106-114. doi:10.1016/j. ejca.2017.09.003

34. Ling S, Song L, Fan N, et al. Combination of metformin and sorafenib suppresses proliferation and induces autophagy of hepatocellular carcinoma via targeting the mTOR pathway. Int $J$ Oncol. 2017;50:297-309. doi:10.3892/ijo.2016.3799

35. Shen C, Peng C, Shen B, et al. Sirolimus and metformin synergistically inhibit hepatocellular carcinoma cell proliferation and improve long-term survival in patients with HCC related to hepatitis B virus induced cirrhosis after liver transplantation. Oncotarget. 2016;7 (38):62647-62656. doi:10.18632/oncotarget.11591

36. Schulte L, Scheiner B, Voigtländer T, et al. Treatment with metformin is associated with a prolonged survival in patients with hepatocellular carcinoma. Liver Int. 2019;39:714-726. doi:10.1111/liv.14048

37. Tseng $\mathrm{CH}$. Metformin and risk of hepatocellular carcinoma in patients with type 2 diabetes. Liver Int. 2018;38:2018-2027. doi:10.1111/liv.13872

38. Kang WH, Tak E, Hwang S, et al. Metformin-associated chemopreventive effects on recurrence after hepatic resection of hepatocellular carcinoma: from in vitro to a clinical study. Anticancer Res. 2018;38:2399-2407. doi:10.21873/anticanres.12490

39. Wang P, Tan G, Zhu M, Li W, Zhai B, Sun X. Hand-foot skin reaction is a beneficial indicator of sorafenib therapy for patients with hepatocellular carcinoma: a systemic review and meta-analysis. Expert Rev Gastroenterol Hepatol. 2018;12:1-8. doi:10.1080/ 17474124.2017.1373018

40. Bruix J, Raoul JL, Sherman M, et al. Efficacy and safety of sorafenib in patients with advanced hepatocellular carcinoma: subanalyses of a phase III trial. $J$ Hepatol. 2012;57:821-829. doi:10.1016/j. jhep.2012.06.014 


\section{Publish your work in this journal}

The Journal of Hepatocellular Carcinoma is an international, peerreviewed, open access journal that offers a platform for the dissemination and study of clinical, translational and basic research findings in this rapidly developing field. Development in areas including, but not limited to, epidemiology, vaccination, hepatitis therapy, pathology

and molecular tumor classification and prognostication are all considered for publication. The manuscript management system is completely online and includes a very quick and fair peer-review system, which is all easy to use. Visit http://www.dovepress.com/ testimonials.php to read real quotes from published authors.

Submit your manuscript here: https://www.dovepress.com/journal-of-hepatocellular-carcinoma-journal 